EPJ Web of Conferences 84, 01004 (2015)

DOI: $10.1051 /$ epjconf/20158401004

(C) Owned by the authors, published by EDP Sciences, 2015

\title{
DESIREE: Physics with cold stored ion beams
}

\author{
R.D. Thomas ${ }^{1, a}$, H.T. Schmidt ${ }^{1}$, M. Gatchell ${ }^{1}$, S. Rosén ${ }^{1}$, P. Reinhed ${ }^{1}$, P. Löfgren ${ }^{1}$, L. Brännholm ${ }^{1}$, \\ M. Blom ${ }^{1}$, M. Björkhage ${ }^{1}$, E. Bäckström ${ }^{1}$, J.D. Alexander ${ }^{1}$, S. Leontein ${ }^{1}$, D. Hanstorp ${ }^{2}$, H. Zettergren ${ }^{1}$, \\ M. Kaminska ${ }^{1}$, R. Nascimento ${ }^{1}$, L. Liljeby ${ }^{1}$, A. Källberg ${ }^{1}$, A. Simonsson ${ }^{1}$, F. Hellberg ${ }^{1}$, S. Mannervik ${ }^{1}$, \\ M. Larsson ${ }^{1}$, W.D. Geppert ${ }^{1}$, K.G. Rensfelt ${ }^{1}$, A. Paál ${ }^{1}$, M. Masuda ${ }^{1}$, P. Halldén ${ }^{1}$, G. Andler ${ }^{1}$, \\ M.H. Stockett ${ }^{1}$, T. Chen ${ }^{1}$, G. Källersjö ${ }^{1}$, J. Weimer ${ }^{1}$, K. Hansen ${ }^{2}$, H. Hartman ${ }^{3,4}$ and H. Cederquist ${ }^{1}$ \\ ${ }^{1}$ Department of Physics, Stockholm University, 10691 Stockholm, Sweden \\ ${ }^{2}$ Department of Physics, University of Gothenburg, 41296 Gothenburg, Sweden \\ ${ }^{3}$ Applied Mathematics and Material Science, Malmö University, 20506 Malmö, Sweden \\ ${ }^{4}$ Lund Observatory, Lund University, 22100 Lund, Sweden
}

\begin{abstract}
Here we will briefly describe the commissioning of the Double ElectroStatic Ion Ring ExpEriment (DESIREE) facility at Stockholm University, Sweden. This device uses purely electrostatic focussing and deflection elements and allows ion beams of opposite charge to be confined under extreme high vacuum and cryogenic conditions in separate "rings" and then merged over a common straight section. This apparatus allows for studies of interactions between cations and anions at very low and well-defined centre-of-mass energies (down to a few $\mathrm{meV}$ ) and at very low internal temperatures (down to a few $\mathrm{K}$ ).
\end{abstract}

\section{Introduction}

One driving force for initial development of ion storage rings was from high-energy particle physics. In recent decades these devices have also proven successful in addressing the needs of the atomic and molecular physics communities into low-energy processes. Many such devices are modeled after LEAR [1] at CERN, in which magnetic elements such as dipole, quadrupole and higher multipole magnets are used to define and control the ion orbits in the device, and examples of such devices are CRYRING [2] (Stockholm, Sweden), ASTRID [3] (Aarhus, Denmark), and TSR [4] (Heidelberg, Germany).

Electrostatic elements are rarely used in these devices: when they are it is primarily for electrostatic septa for the injection/extraction of ions. This aspect is relevant when it comes to comparing devices based on electrostatic storage as opposed to magnetic storage. Using electrostatic elements clearly removes the problems associated with effects such as remanent fields and hysteresis in the magnets and the requirements for water cooling. Magnets are also expensive, big and heavy, while with electrostatic elements a compact and less expensive solution can be realized. The fact that all elements are electrostatic means that, for an injector on a given potential, the mass and charge of the injected ions can be changed without needing to change the settings of the ion-optical elements. Another advantage is

\footnotetext{
${ }^{a}$ Corresponding author: rdt@fysik.su.se
}

This is an Open Access article distributed under the terms of the Creative Commons Attribution License 4.0, which permits unrestricted use, distribution, and reproduction in any medium, provided the original work is properly cited. 
that an electrostatic device can store heavy ions in low charge states, something which is often a problem in magnetic rings where the maximum mass of the stored ions is limited by the bending power of the magnets, and this plays a large role in the experimental uncertainties [5]. Finally, also from a purely scientific perspective, the lack of magnetic fields also plays a role in studied of fundamental physics since such fields can give rise to mixing and transitions between quantum states in the ions being stored in the device.

These advantages were realised through the pioneering work of Søren Pape Møller in Aarhus, Denmark, with the construction of the purely electrostatic storage ring ELISA [5], and since ELISA was constructed several new electrostatic storage rings have been built or are under construction around the world, for examples in Japan [6, 7], Frankfurt [9], Saudi Arabia [8]. It is noted here that one of the rings constructed in Japan [7] is cooled down to liquid nitrogen temperatures and operates under these cyrogenic conditions.

The cryogenic aspect leads to two of the more ambitious of the "next generation" of electrostatic storage rings, both of which are planned to operate at significantly sub LN2 temperatures, i.e. $<20 \mathrm{~K}$. One of these facilities currently being constructed at the Max Plank Institute for Nuclear Physics, Heidelberg, Germany, is a $35 \mathrm{~m}$ circumference single cryogenic electrostatic ion-storage ring (CSR), and which will be equipped with an internal gas-jet target, an ion-neutral merging section, and an electron cooler/target device [10]. The current status of this facility is also discussed in this book of abstracts. The second is the subject of this presentation: the Double ElectroStatic Ion-Ring ExpEriment, DESIREE, currently under construction at Stockholm University, Sweden. A technical article focussed on the construction of DESIREE is available [11], and the results from the initial commissioning experiments has been recently Spublished [12]. The primary objective of DESIREE is to study interactions between $\mathrm{keV}$ beams of positive and negative ions at very low and well controlled center-of-mass energies (down to a few $\mathrm{meV}$ ) and at very low internal temperatures (down to a few $\mathrm{K}$ ).

\section{The DESIREE facility}

Figure 1 shows a schematic overview of the DESIREE facility: The main DESIREE vacuum vessel, the two ion source platforms, and the main ion beamlines to inject the ions into the storage rings.

A detailed description of the construction of DESIREE is available [11], so is only briefly discussed here. The main DESIREE vessel consists of a double-walled chamber, i.e., two separate vacuum vessels with one placed inside the other and separated by a thermal screen. The outer box is constructed entirely of steel plates which are welded together. For mainly thermal-conduction reasons the inner chamber is constructed welded from plates of a particular aluminium alloy. The latter was chosen becuase of being relatively easily machinable, and because its sufficiently good thermal conductance and known thermal properties over the whole temperature range from $400 \mathrm{~K}$ down to a few Kelvins. All ion-optical elements and detectors are carefully mounted directly on the bottom of this chamber, and access to all components for their mounting is through the top of the chamber via a removable lid. AstraSeal gaskets are used for all sealing surfaces. The two vessels are separated by a thermal screen which, for its excellent thermal conductivity, is constructed from copper sheets and 30 layers of Mylar super-insulation which are placed directly on the outside of the copper screen.

Figure 2 shows a rough schematic of the ion-optical layout of the inner chamber, the heart of the DESIREE facility.

\subsection{The Rings}

Each ring is $\approx 8.8 \mathrm{~m}$ in circumference, and has two $160^{\circ}$ cylindrical deflectors and four $10^{\circ}$ parallelplate horizontal deflectors, where the two $10^{\circ}$ deflectors on both ends of the merging section (MR) are common to both rings. Only ions with opposite charge can be stored if both rings are operated at the 


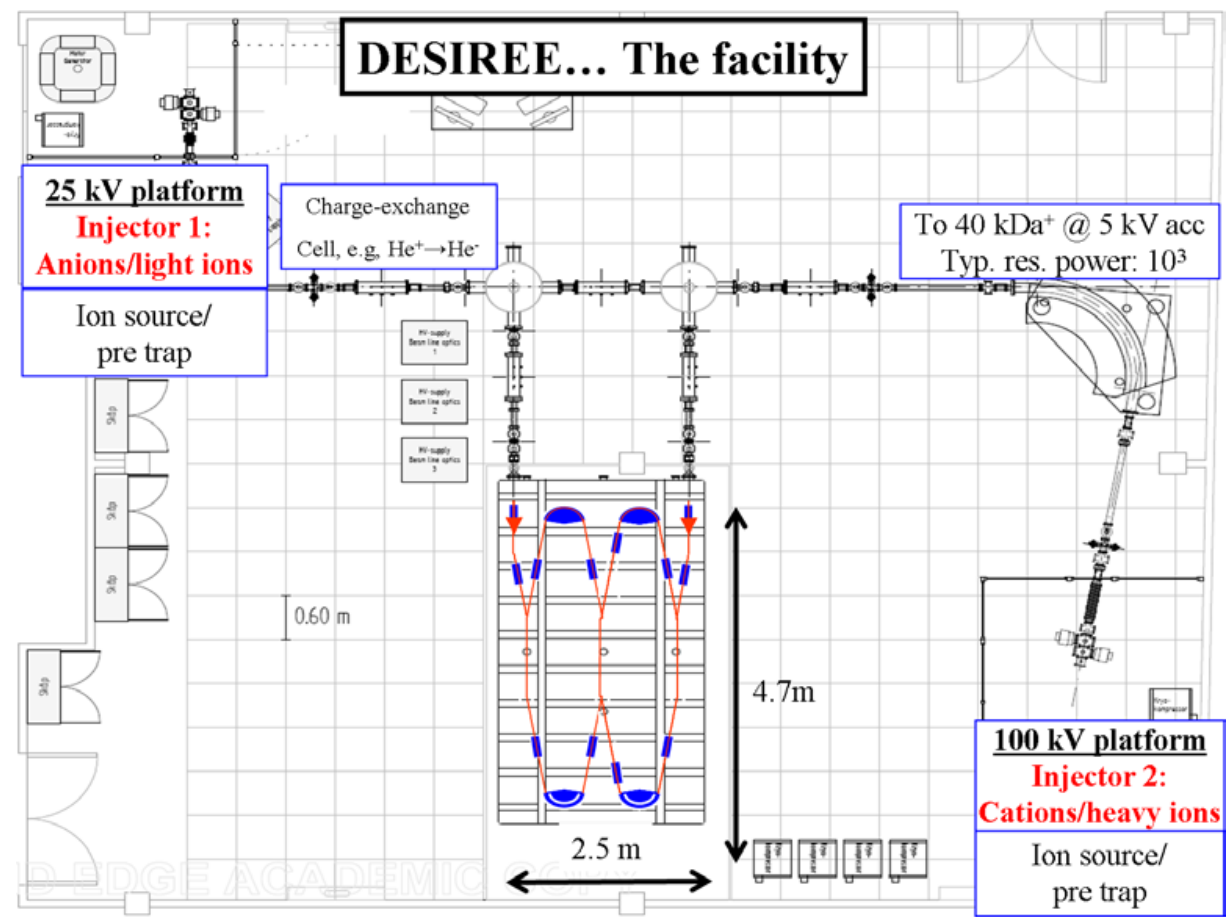

Figure 1. A schematic overview of the DESIREE facility, showing the main DESIREE vacuum vessel, the two ion source platforms, and the main ion beamlines.

same time, though either ring can be operated independently with ions of any charge. Ring 1 is the defining ring, i.e., the voltage on the $10^{\circ}$ deflector after the common merging section is defined such that the ions in Ring 1 will be always be correctly deflected into the correct orbit. As such, Ring 2 (R2) has a somewhat different ion-optical layout compared to Ring 1 (R1), and can store ions with different energy [and charge] compared to R1, i.e. these ions require a different deflection voltage to be correctly stored and so to compensate, and to make the ion beams collinear in the merging region, additional deflector plates are added to R2.

\subsection{Cryogenic operation}

During normal cryogenic operation, the inner chamber is cooled with four cryogenerators (Sumitomo RDK-415D) where the first stage of each cryogenerator is connected to the thermal screen and the second stage to the bottom of the inner chamber. The total heat load on the thermal screen and the inner vessel is estimated to be $\approx 140 \mathrm{~W}$ and $\approx 9 \mathrm{~W}$, respectively. At cryogenic temperatures the inner vessel will be pumped by the cold walls of the chamber. The main contribution to the background pressure will be $\mathrm{H}_{2}$, which is reduced by utilising Ti-sublimation pumping. After the initial baking at $400 \mathrm{~K}$ of the aluminium vessel, a pressure of $1 \times 10^{-10}$ mbar was reached at room temperature. Figure 3 illustrates the cooldown time for DESIREE, i.e. it takes almost two weeks to cool down from $\approx 300 \mathrm{~K}$ to $\approx 15 \mathrm{~K}$.

\subsection{Delivering ions}

In addition to the "traditional" filament and cold-cathode ion sources which have been used to create the singly charged atomic and molecular ions studied in molecular storage rings such as CRYRING, 


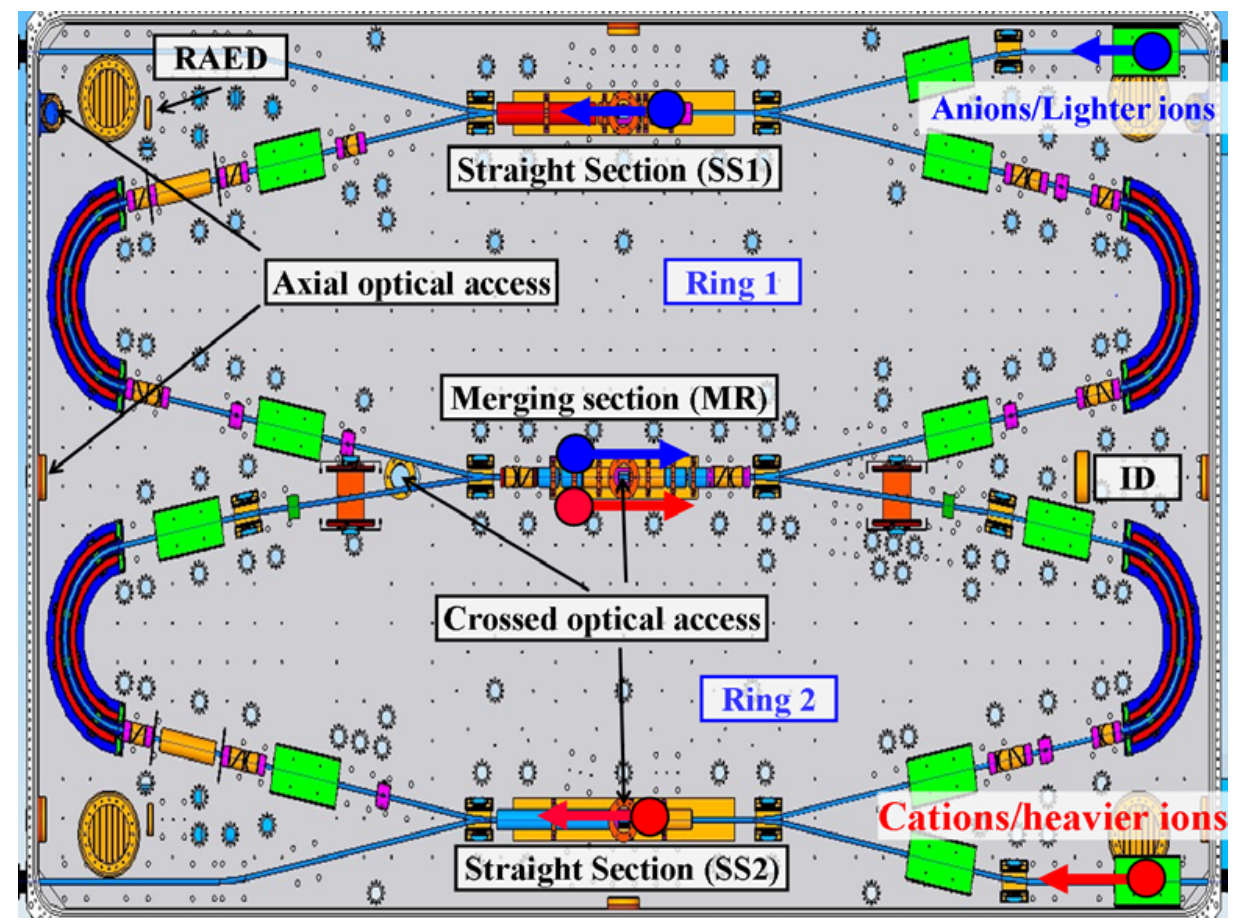

Figure 2. A rough schematic of the heart of the DESIREE facility showing: the two rings, the two straight sections in each ring (SS1,SS2) and the common straight section - the merging section MR. The position of several of the available particle detectors: the imaging detector (ID) and a resistive anode encoder (RAED), are noted, as are viewports for laser access allowing co-axial and crossed laser-ion beam interactions.

\section{DESIREE cooldown}

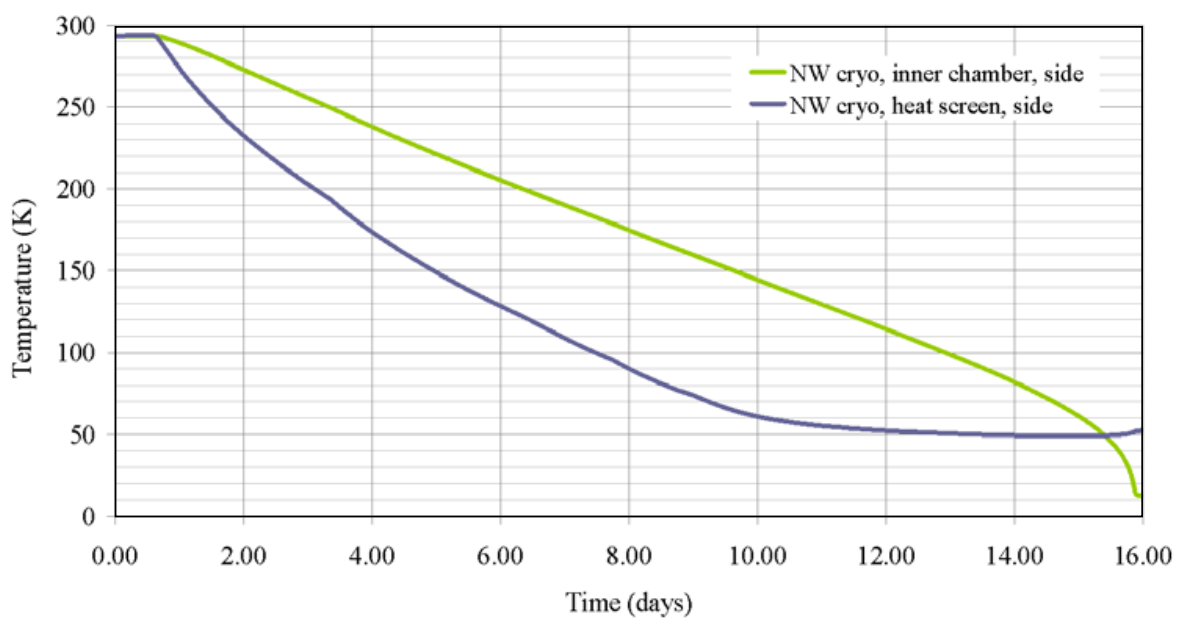

Figure 3. An example cooling curve for the inner chamber and thermal screen in DESIREE. 
for examples in studies of Dissociative Recombination [13, 14], the platforms have been designed to accommodate a suite of different ion sources ranging from an expansion source for producing rotationally cooled molecular ions (see e.g. [15]), an electrospray source for creating biomolecules and large molecular cluster ions, and a sputter source for generating negative ions (see e.g. [16]), where the latter is currently used to generate the $\mathrm{C}^{-}$ion discussed later [12].

\subsection{Development phase}

It became quickly clear during the initial development of the project that in order to meet the technical challenge of designing a complex system like DESIREE a smaller test and training system needed to be constructed. This chamber was built as a cryostat, with an inner and outer chamber and a copper screen in between, and represented an almost exact copy of the design for DESIREE. One of the important tasks for this chamber was to test the cryogenic properties of the different kinds of detectors that were planned for use in DESIREE. One such family of detectors are microchannel-plate based detectors (MCPs), in which these plates are coupled to different types of anode depending on the purpose of the detector. Both resistive- and phosphor screen-anodes have been tested, and the results from the MCP-phosphor screen anode have been reported [17], and showed that these detectors successfully operate under cryogenic conditions. Both detector types will be used in DESIREE: resistive-anode and phosphor-anode and these are indicated as RAED and ID in Fig. 2, respectively.

One of the most significant scientific highlights to come out of these investigations arose from the placing of an electrostatic ion trap in the test chamber, i.e., to mimic the ion-storage properties of DESIREE. Here, a linear ion-trap, ConeTrap [18], was placed inside the inner vacuum chamber. A pulse of ions was injected into the trap, which was then closed, and the lifetime of the ions in the trap studied as function of their storage time. The ions chosen for this particular test were the metastable helium anion, $\mathrm{He}^{-}\left(1 \mathrm{~s} 2 \mathrm{~s} 2 \mathrm{p}{ }^{4} \mathrm{P}_{5 / 2}\right)$, with the motivation that results from earlier lifetime measurements were limited in the accuracy of systematic effects due to the photo-detachment of the loosely bound $2 \mathrm{p}$ electron by $300 \mathrm{~K}$ blackbody radiation photons emitted from the surrounding vacuum chamber. In performing this experiment in a suitable cryogenic environment, in practice less than $80 \mathrm{~K}$, this effect is eliminated. The results from these tests gave the most accurate measurement for the lifetime of these ions [19], as well as providing valuable information on the ion-beam storage lifetimes as a function of the residual gas pressure in the chamber [20].

\section{Commissioning and illustrative research ideas}

DESIREE has been planned and constructed so that any given experiment can be undertaken in either of the two rings or a single experiment can utilise both rings. Examples of such experiments are discussed in the recently published technical paper [11], as well as the first commissioning paper [12]. The most unique feature is the merging region, and so we briefly discuss an example of the type of experiment which now can be undertaken at DESIREE and which otherwise have proven extremely difficult or impossible.

The possibility to perform merged-beams experiments with positive and negative ions that are stored and cooled to low temperatures by temperature equilibrium with the surroundings is the most clearly unique feature of DESIREE. Consider the simplest mutual neutralization $(\mathrm{MN})$ process between small atomic cations and anions: $\mathrm{A}^{+}+\mathrm{B}^{-} \rightarrow \mathrm{A}+\mathrm{B}+E_{R}$. This is a single electron transfer process, and the available reaction energy, $E_{R}$, is given to the fragments as electronic excitation and kinetic energy. Naturally, any energy used to electronically excite the fragments means less kinetic energy is given to the two products. Thus MN is an ionization reducing process. The formation of polyatomic ions in the interstellar medium is considered to be through chemistry involving ionized species. The degree of ionization in the interstellar clouds is determined by a balance between ionization through cosmic 


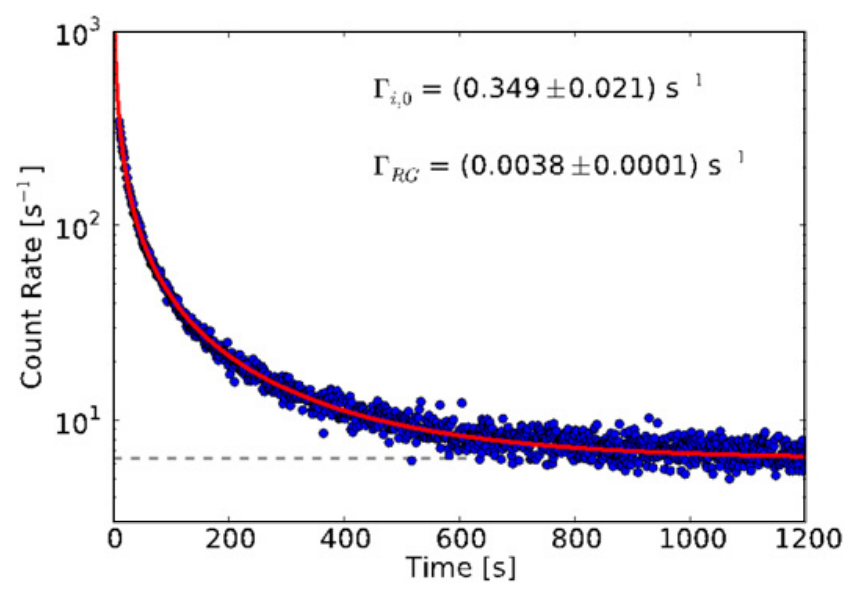

Figure 4. Typical storage lifetimes for a $10 \mathrm{keV} \mathrm{C}^{-}$ion beam in Ring 1.

and stellar radiation and neutralization processes. In the gas phase two such neutralization processes exist: Electron-cation dissociative recombination (DR) and cation/anion mutual neutralization (MN). One of the primary motivations for the studies at CRYRING of DR for astrophysically abundant cations $[13,14]$ has been the role of this process in interstellar chemistry in regions where the negative charge is primarily in the form of free electrons. One of the most recent observations in the interstellar medium has been that of long carbon-chain anions, the first of these only unambiguously identified less than 10 years ago [21]. If present in the amounts required to explain the observed absorption, it is possible that in these regions negative charge is more often in the form of anions than free electrons. In such an environment the role of MN in the ion chemistry is more important than DR and the possibility offered by DESIREE to study these processes highly relevant to this field.

The simplest carbon anion is $\mathrm{C}^{-}$, and during the commissioning phase of DESIREE this ion has been used extensively. One unique aspect of DESIREE is the cryogenic environment, and the cryo-pumping that this provides. Studying the storage properties of ions in DESIREE allows us to calculate the numberdensity of residual gas particles inside the inner chamber if the interaction of the ions with these particles determines the storage lifetime. This is a parameter which is almost impossible to determine otherwise due to the inability of even modern pressure gauges to measure under such ultra hard vacuum conditions. Figure 4 plots a typical lifetime curve for $\mathrm{C}^{-}$, obtained by measuring the neutral carbon atoms created when, e.g., $\mathrm{C}^{-}$undergoes collisional detachment with a residual gas particle.

The residual gas number density is sufficiently low that collisions are infrequent and ions can be stored for 100s of seconds. If the lifetime is determined by just such collisions, it is possible to determine the number density of residual gas particles in the storage ring itself. Analysis of the storage lifetime data for $\mathrm{C}^{-}$under a variety of conditions, as well investigating other reaction processes which involve interaction with the residual gas particles, allows us to determine that the residual gas number density is on the order $2-5 \times 10^{4} \mathrm{~cm}^{-3}$, which corresponds to a pressure, $p=4 \times 10^{-14}$ mbar at $13 \mathrm{~K}$ [12].

\section{Current Status}

The final states of commissioning are underway. Baseline measurements to determine, e.g., the pressure and residual gas number density in the ring, as well as absolute numbers of stored ions are almost completed. During the next few months, e.g. Dec 2013-Mar 2014, the detectors, and the data acquisition \& analysis components necessary to study MN reactions with full multiple-particle coincidence will 
be implemented. Finally, several further papers will be written from data taken during experiments conducted under the commissioning period.

\section{References}

[1] T. Walcher, Ann. Rev. Nucl. Part. Sci. 38 (1988) 67

[2] K. Abrahamsson et al. Nucl. Instrum. Methods Phys. Res., Sect. B 79 (1993) 269

[3] S. P. Møller, in Conference Record of the 1991 IEEE Particle Accelerator Conference, edited by L. Lizama and J. Chew (New York, IEEE, 1991) (1991) 2811

[4] D. Krämer et al., Nucl. Instrum. Methods Phys. Res. A 287 (1989) 268

[5] S. P. Møller, Nucl. Instrum. Methods Phys. Res. A 394 (1997) 281

[6] T. Tanabe et al., Phys. Rev. Lett. 90 (2003) 193201

[7] S. Jinno et al., Nucl. Instrum. Methods Phys. Res. A 532 (2004) 477

[8] M. E. Ghazaly et al., Nucl. Instrum. Methods Phys. Res. A 709 (2013) 76

[9] K. Stiebing et al., Nucl. Instrum. Methods Phys. Res. A 614 (2010) 10

[10] R. von Hahn et al., Nucl. Instrum. Methods Phys. Res. B 269 (2011) 2871

[11] R. Thomas et al., Rev. Sci. Instrum. 82 (2011) 065112

[12] H. T. Schmidt et al. Rev. Sci. Instrum. 84 (2013) 055115

[13] R. D. Thomas, Mass. Spec. Rev. 27 (2008) 485

[14] M. Larsson and A. E. Orel, Dissociative recombination of molecular ions (Cambridge University Press, Cambridge, 2008)

[15] H. Kreckel et al., Phys. Rev. A 82 (2010) 042715

[16] A. Lindahl et al., Phys. Rev. A 77 (2008) 022710

[17] S. Rosén et al. Rev. Sci. Instrum. 78 (2007) 113301

[18] H. T. Schmidt et al., Nucl. Instr. Methods Phys. Res. B 173 (2001) 523

[19] P. Reinhed et al., Phys. Rev. Lett. 103 (2009) 213002

[20] P. Reinhed et al., Nucl. Instrum. Methods Phys. Res. A 621 (2010) 83

[21] M. C. McCarthy et al., Ap. J. 652 (2006) L141 